\title{
Not for adults only: MitraClip use in a paediatric patient
}

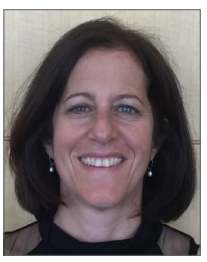

Denise C. Joffe ${ }^{1}$, MD; Thomas K. Jones ${ }^{2}$, MD; Mark Reisman³, MD, FASE;

Elizabeth Perpetua ${ }^{4}$, ARNP; Yuk Law ${ }^{2}$, MD; Andreas Schuler ${ }^{1}$, MD;

G. Burkhard Mackensen ${ }^{1 *}, \mathrm{MD}, \mathrm{PhD}, \mathrm{FASE}$

1. Division of Cardiothoracic Anesthesiology and Critical Care Medicine, Department of Anesthesiology and Pain Medicine, University of Washington Medical Center, Seattle, WA, USA; 2. Division of Cardiology, Department of Pediatrics, Seattle Children's Hospital, Seattle, WA, USA; 3. Division of Interventional Cardiology, Department of Cardiology, University of Washington Medical Center, Seattle, WA, USA; 4. Division of Cardiothoracic Surgery, Department of Surgery, University of Washington Medical Center, Seattle, WA, USA

This paper also includes supplementary data published online at: http://www.pcronline.com/eurointervention/105th_issue/172

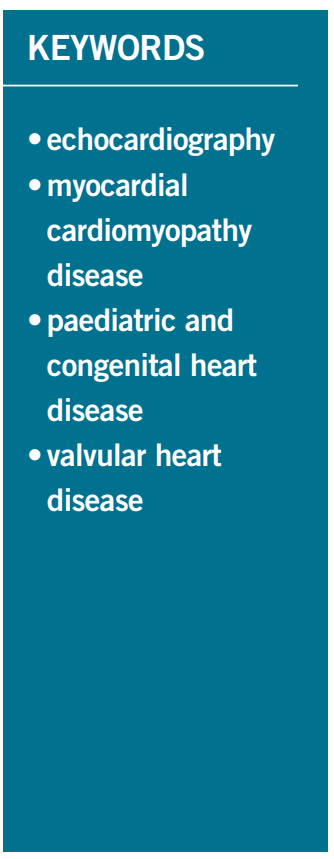

\section{Abstract}

We present the primary report of a paediatric patient who had placement of the MitraClip device for severe functional mitral regurgitation. The patient was a 14-year-old boy with symptomatic end-stage non-compaction type cardiomyopathy secondary to a mitochondrial cytopathy. He had severe mitral regurgitation, tricuspid valve regurgitation, a severely dilated LV with apical non-compaction, severe LV dysfunction and severe pulmonary hypertension. Despite optimal medical therapy he developed progressive symptoms of congestive heart failure and he was not a candidate for an assist device or cardiac transplantation. Multimodality imaging with fluoroscopy, two-dimensional and three-dimensional transoesophageal echocardiography was used to guide the procedure. Two MitraClips were placed resulting in an adequate decrease in MR severity to no more than mild-moderate. Five months post MitraClip implantation, he has improved exercise tolerance. Transthoracic echocardiography showed mild to moderate mitral regurgitation and a decrease in left ventricular size. To our knowledge, this is the first reported paediatric patient to be offered this innovative procedure. Percutaneous edge-to-edge MV repair may prove to be a novel and effective palliation to consider in a subgroup of paediatric, adolescent and young adult patients. This case report describes some of the considerations specific to the paediatric patient.

*Corresponding author: Department of Anesthesiology and Pain Medicine, University of Washington Medical Center, 1959 N.E. Pacific Street, Seattle,WA 98195,USA.E-mail: gbmac@uw.edu 


$\begin{array}{ll}\text { Abbreviations } \\ \text { CFD } & \text { colour flow Doppler } \\ \text { LAX } & \text { long-axis } \\ \text { ME } & \text { mid oesophageal } \\ \text { MPR } & \text { multiplanar reconstruction } \\ \text { SAX } & \text { short-axis } \\ \text { TG } & \text { transgastric }\end{array}$

\section{Background}

Percutaneous edge-to-edge mitral valve (MV) repair performed with the MitraClip ${ }^{\circledR}$ device (Abbott Vascular, Santa Clara, CA, USA) in high-risk or inoperable adult patients with severe symptomatic mitral regurgitation (MR) has been shown to be an effective therapy that reduces symptoms and improves functional status ${ }^{1}$. The safety and effectiveness of MitraClip therapy has not been established in paediatric patients since, in most instances, other more durable and proven treatment choices such as MV surgery (repair or replacement) or heart transplantation are available to them. Here we present a paediatric patient with heart failure symptoms in conjunction with severe functional MR (FMR) who had successful placement of the MitraClip device.

\section{Case report}

The case was a 14-year-old, $50.6 \mathrm{~kg}$ (BSA $1.47 \mathrm{~m}^{2}$ ) boy with severe neurodevelopmental delay and symptomatic end-stage dilated and non-compaction type cardiomyopathy secondary to a mitochondrial cytopathy involving electron transport chain complexes 1 and 4. Despite optimal chronic medical therapy (lisinopril, carvedilol, spironolactone, furosemide) which could not be increased due to hypotension, he developed progressive symptoms of congestive heart failure (CHF).
A pre-procedure transthoracic echocardiogram (TTE) showed a left ventricular ejection fraction (LVEF) of 35-40\%, severe central MR, a markedly enlarged left atrium (LA), severe pulmonary hypertension (PHTN) with suprasystemic pulmonary artery pressures (PAP), moderate tricuspid valve regurgitation (TR) and a mild decrease in right ventricular (RV) function. The family declined evaluation for cardiac transplantation but was interested in pursuing percutaneous MV therapy. He was referred for consideration of percutaneous MV repair with the MitraClip device.

Following thorough consultation with all members of the Heart Valve Team and informed consent, percutaneous edge-to-edge repair of his MV was undertaken. A paediatric interventional cardiologist, and paediatric and adult cardiac anaesthesiologist assisted with his care and procedural imaging. A significant amount of preoperative planning was undertaken to care for a child in a hospital that serves adult patients. His unique psychosocial needs as well as the anaesthetic management of his cytopathy were the major differences in his management compared to a typical patient receiving MitraClip therapy.

Multimodality imaging with fluoroscopy and two-dimensional (2D) as well as three-dimensional (3D) transoesophageal echocardiography (TEE) was used to guide the procedure (iE33; Philips Medical Systems, Andover, MA, USA). The pre-procedure TEE exam showed a severely dilated LV with apical non-compaction, severe LV dysfunction and severe MR along the entire coaptation line (Figure 1, Figure 2, Moving image 1, Moving image 2).

Based on 3D quantification, the MV annulus was enlarged at $3.7 \times 3.6 \mathrm{~cm}$ and the vena contracta area (VCA) measured $0.86 \mathrm{~cm}^{2}$ (Figure 3). 3D colour imaging further elucidated that there were two distinctive MR jets along the coaptation line, a larger medial jet and a smaller more lateral jet (Figure 4, Moving image 3).

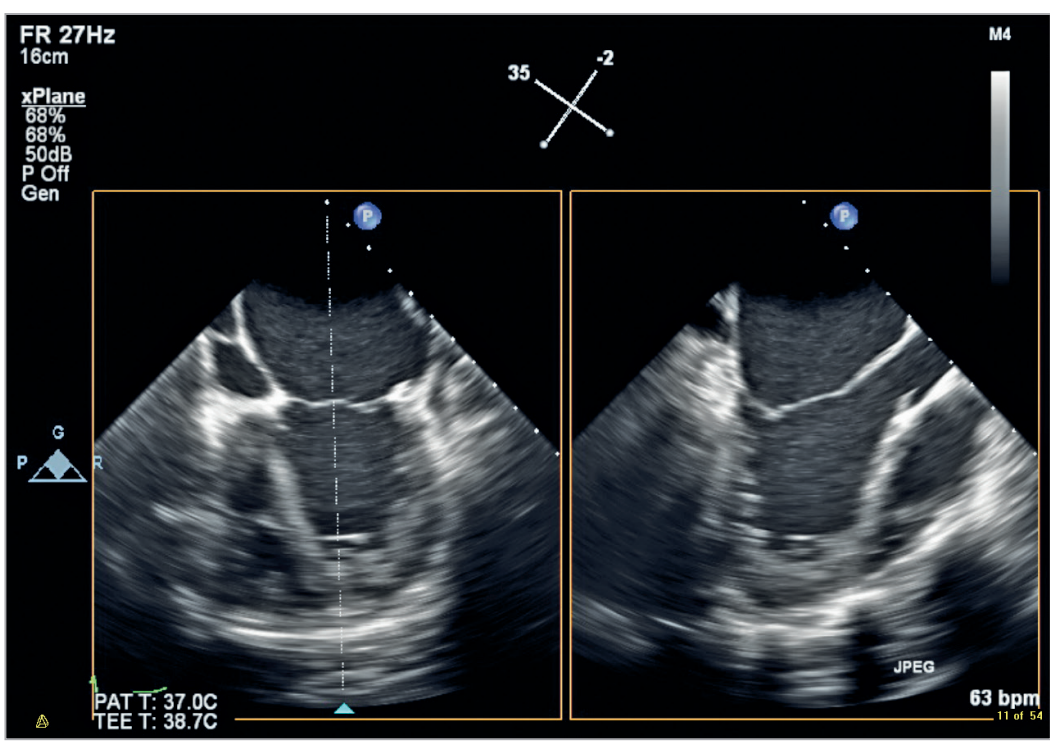

Figure 1. ME biplane acquisition showing a modified four-chamber view on the left and a perpendicular LAX view of the LV on the right. Note the enlarged left atrium and the globular LV with apical non-compaction. The MV leaflets are apically tethered during systole and have little opportunity for coaptation. 


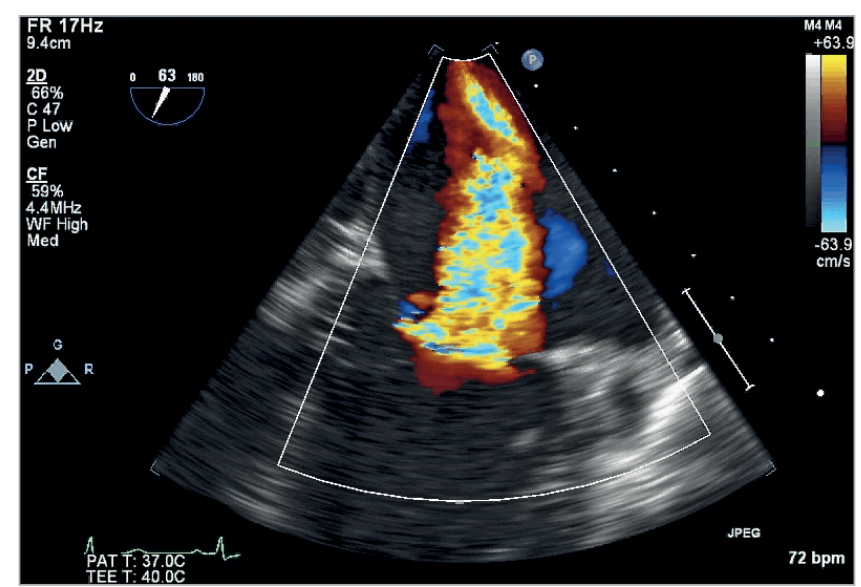

Figure 2. 2D CFD ME commissural view of the $M V$ showing severe $M R$ along the entire coaptation line.

Given the comprehensive echocardiographic assessment at the beginning of the case, the treatment strategy included a high likelihood of requiring two MitraClips to obtain a significant reduction of MR. Thus, the first clip was deployed in the central portion of the MV (A2/P2 segment) with the intention of placing a second clip medial to the first (Figure 5, Moving image 4). The second clip created a much smaller medial orifice and resulted in a further reduction of MR to mild-moderate (Figure 6, Figure 7, Moving image 5) with an increase in the mean gradient across both orifices to 6-7 $\mathrm{mmHg}$. The net result was a slightly greater mean transmitral gradient than desired but, given the significant decrease in MR, there was a net decrease in his PAP (Table 1).

The patient was extubated immediately post procedure and, after a short stay in the recovery unit, was transferred to the

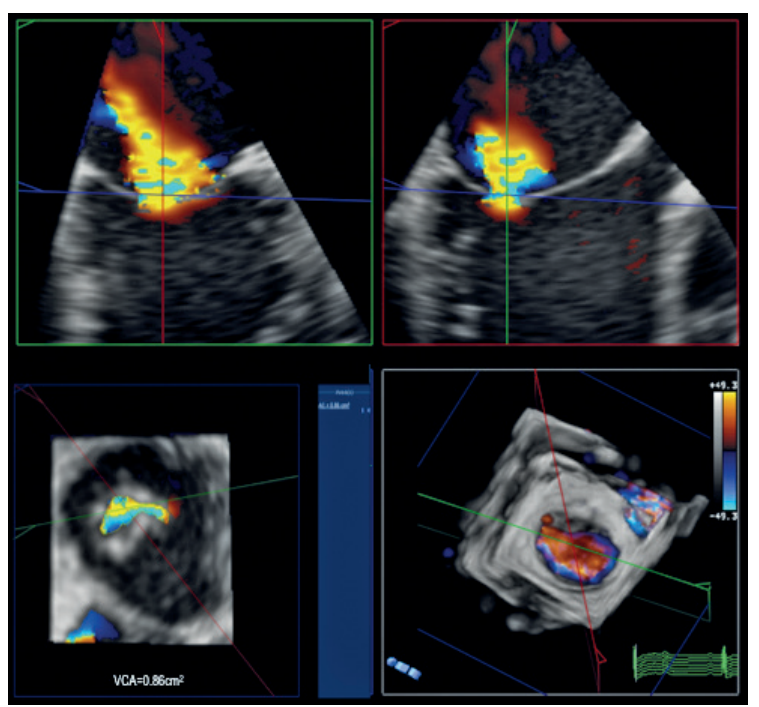

Figure 3. 3D colour MPR of the MR jet before placement of the first MitraClip. The MPRs are accurately aligned to measure the VCA at baseline. Note the elliptical shape of the VCA as typically seen in FMR. At baseline, the VCA measured $0.86 \mathrm{~cm}^{2}$.

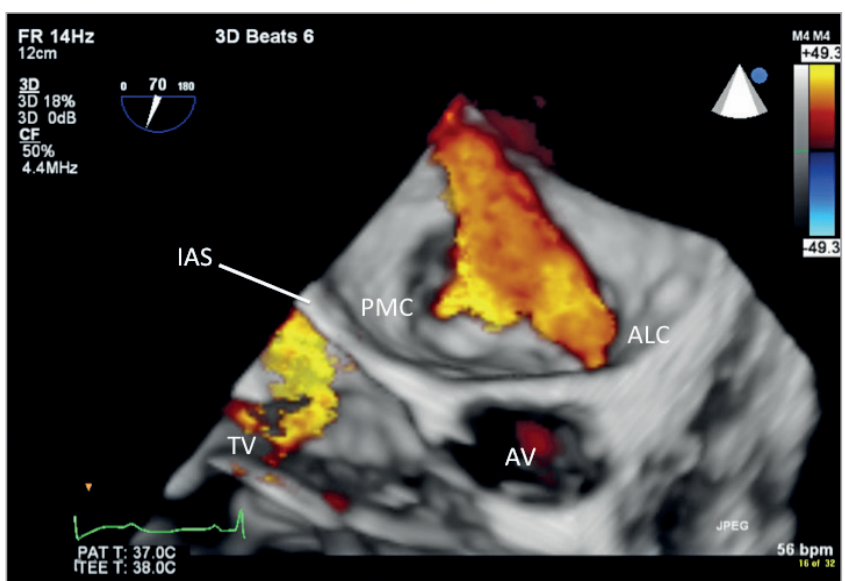

Figure 4. $3 D$ colour image of the $M V$ with the posteromedial commissure (PMC) on the left and the anterolateral commissure (ALC) towards the right. Unidirectional colour selection was used to demonstrate only regurgitant flow while removing the colour distraction created by mitral inflow. Note that, compared to the orthogonal 2D colour images (Figure 2, Moving image 2A, Moving image $2 B$ ), $3 D$ colour is extremely useful in providing details of the geometry of the MR. This image also shows the aortic valve in front $(A V)$ and moderate TR directed towards the interatrial septum (IAS).

ric intensive care unit at Seattle Children's Hospital. He was discharged from hospital four days later.

On his recent follow-up visit, five months post MitraClip therapy, the patient was in satisfactory condition. His TTE showed the two clips in stable position. Compared to the pre-procedure TTE, there was moderate MR, a decrease in left ventricular internal diameter in diastole to $52 \mathrm{~mm}$ from $59.3 \mathrm{~mm}$, a decrease in LA volume index to $62 \mathrm{ml} / \mathrm{m}^{2}$ from $72.2 \mathrm{ml} / \mathrm{m}^{2}$, an improvement in PAP to $3 / 4$ of systemic pressure and an increase in LVEF from $39 \%$ to $41 \%$. The family described improved exercise tolerance and appetite. His brain natriuretic peptide (BNP) is trending downwards.

Table 1. Intra-procedure haemodynamics.

\begin{tabular}{|c|c|c|c|}
\hline $\begin{array}{c}\text { Haemodynamic/echo } \\
\text { parameters }\end{array}$ & $\begin{array}{l}\text { Pre MitraClip } \\
\text { in OR }\end{array}$ & $\begin{array}{l}\text { Post } 1^{\text {st }} \\
\text { MitraClip }\end{array}$ & $\begin{array}{l}\text { Post } 2^{\text {nd }} \\
\text { MitraClip }\end{array}$ \\
\hline HR (beats per minute) & 71 & 72 & 70 \\
\hline $\mathrm{BP}(\mathrm{mmHg})$ & $75 / 50(58)$ & $90 / 52(65)$ & $100 / 55(70)$ \\
\hline CVP (mmHg) & 10 & 15 & 12 \\
\hline PAS (mmHg) & 70 & 68 & 62 \\
\hline PAD (mmHg) & 40 & 27 & 24 \\
\hline mPAP (mmHg) & 50 & 41 & 37 \\
\hline LAP (mmHg) & 22 v wave 35 & 18 v wave 30 & NA \\
\hline TPG (mmHg) & $15-28$ & $11-21$ & \\
\hline TEE mean mitral gradient (mmHg) & No gradient & 3 & 6 \\
\hline \multicolumn{4}{|c|}{$\begin{array}{l}\text { BP: blood pressure; CVP: central venous pressure; HR: heart rate; LAP: left atrial pressure; } \\
\text { mPAP: mean pulmonary artery pressure; NA: not available; OR: operating room; } \\
\text { PAD: pulmonary artery diastolic pressure; PAS: pulmonary artery systolic pressure; } \\
\text { TPG: transpulmonary gradient }\end{array}$} \\
\hline
\end{tabular}




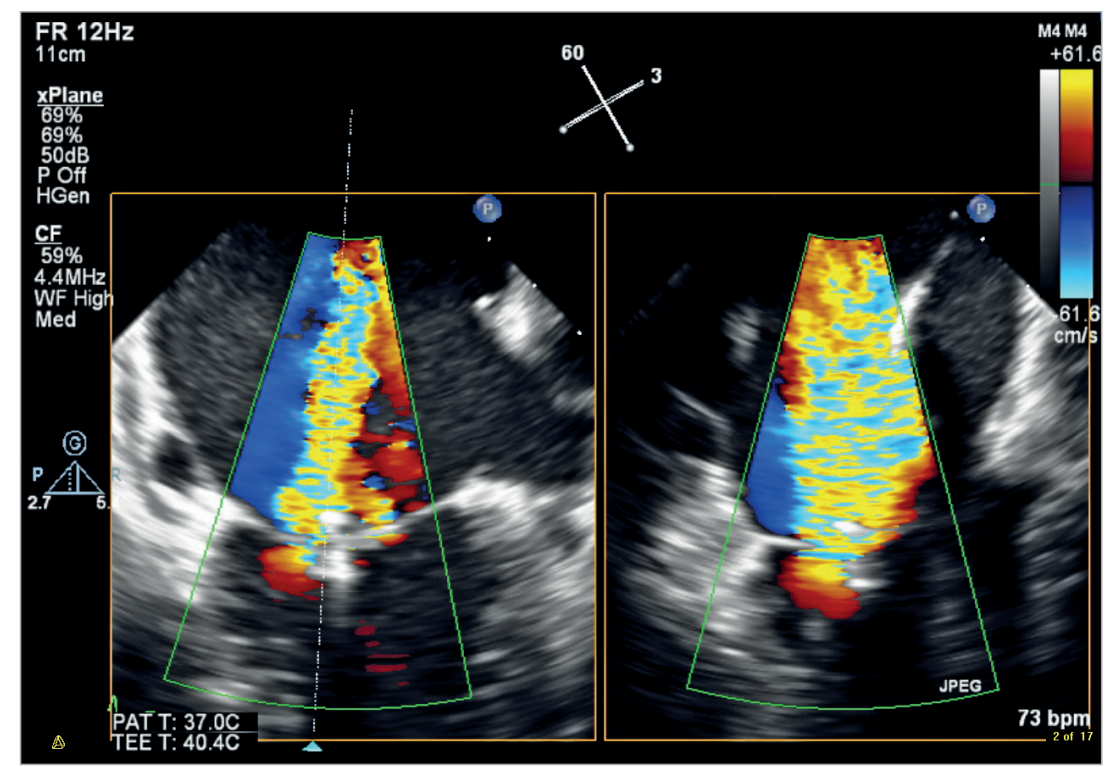

Figure 5. ME CFD biplane image of the MV after deployment of the first MitraClip in the area of A2/P2. MV commissural view on the left and corresponding LAX view of the MV on the right. Note the significant residual MR medial to the first MitraClip.

\section{Discussion}

Paediatric patients with cardiomyopathies secondary to genetic or congenital diseases, such as mitochondrial cytopathies, muscular dystrophies and other metabolic disorders, can present with a wide range of clinical symptoms and prognoses. At the severe end of the spectrum, they may have associated significant comorbidities such as respiratory failure, severe neurocognitive or neuromuscular dysfunction, resulting in limited life expectancy ${ }^{2}$. Once these patients develop symptoms of advanced CHF, few treatment options are offered beyond medical therapy. Mechanical support and heart transplantation are often not considered because life expectancy remains limited by non-cardiac comorbidities.

Percutaneous edge-to-edge MV repair decreases FMR, LV dimensions, and improves symptoms in adults who are not candidates for surgical repair and may prove to be a novel and effective palliation to consider in paediatric patients with limited therapeutic options.

For technical reasons, the patient's cardiovascular anatomy (especially peripheral vessels) needs to be large enough to accommodate the $24 \mathrm{Fr}$ introducer sheaths as well as allowing manoeuvrability of the MitraClip delivery system within the left

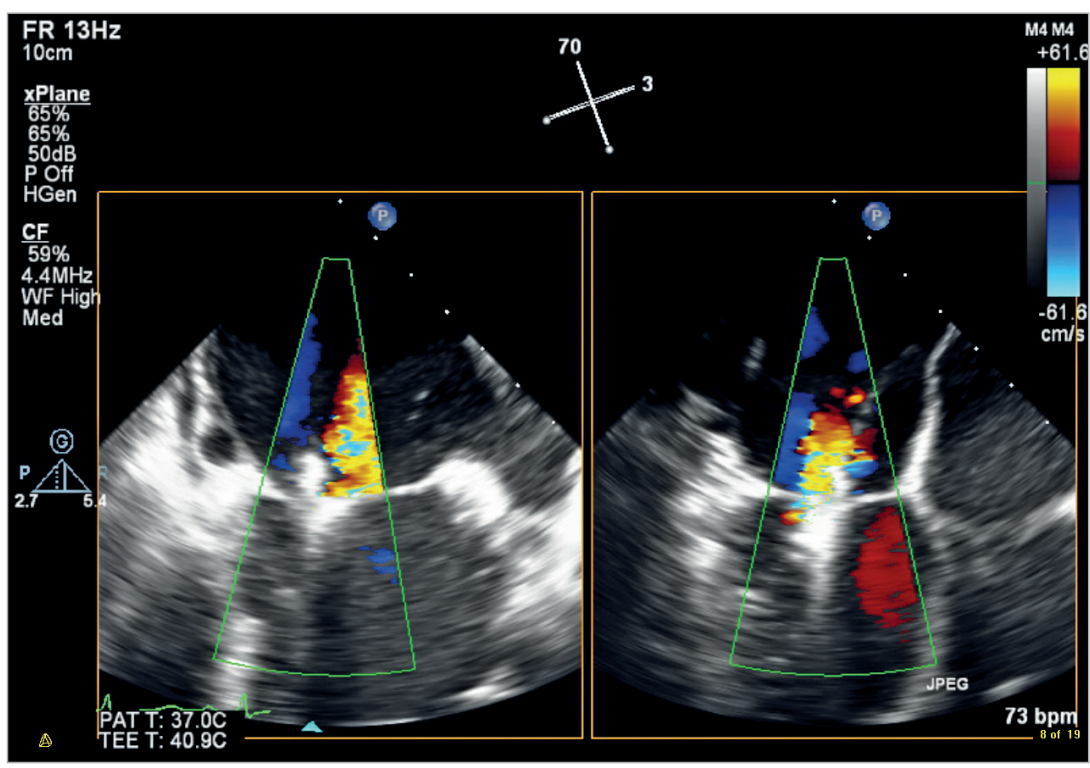

Figure 6. ME colour biplane image displaying the MV in a commissural view and a corresponding LAX view after the second MitraClip has been deployed. The image displays further reduction of MR leaving only mild to moderate residual MR laterally. 


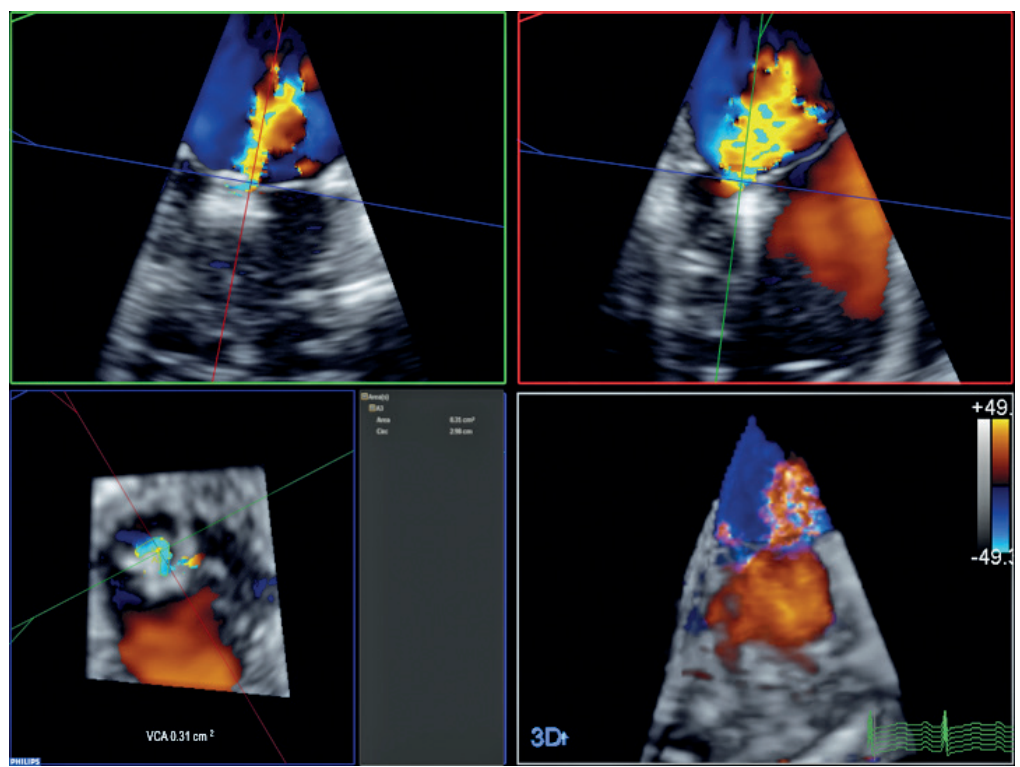

Figure 7. 3D colour MPR of the MR jet following deployment of both MitraClips. The VCA has been significantly reduced to $0.31 \mathrm{~cm}^{2}$ post treatment.

atrium. These requirements correspond to a minimum weight of approximately $40-45 \mathrm{~kg}$ and minimum age of about nine to 12 years. The procedure is performed using the same size transseptal puncture needle, MitraClip and percutaneous closure devices as in adults.

Although there is the concern for an increased risk of creating mitral stenosis in smaller patients, most patients with FMR have a dilated MV annulus and increased LA volumes. In addition, even at the lower age of this range, the annulus is usually more than $85 \%$ of its expected adult size ${ }^{3}$. Finally, there are reports describing surgically placed Alfieri stitches in paediatric patients as young as two years without creating mitral stenosis ${ }^{4}$. Therefore, it seems as though the risk of creating unacceptable mitral stenosis may be less than anticipated based on age.

\section{Conclusion}

To our knowledge, this is the first reported paediatric patient to be offered this innovative procedure for worsening FMR in the setting of a cardiomyopathy. Preliminary results from early followup indicate that percutaneous edge-to-edge MV repair may have a role as compassionate therapy in a subgroup of paediatric, adolescent and young adult patients with advanced FMR.

\section{Impact on daily practice}

This is a primary report on the use of the MitraClip procedure to palliate symptomatic severe functional MR in a paediatric patient with a limited life expectancy. Although the MitraClip procedure was not conceptualised for the paediatric population, this treatment option should be considered as compassionate therapy in a subgroup of paediatric, adolescent and young adult patients with advanced FMR.

\section{Conflict of interest statement}

The authors have no conflicts of interest to declare.

\section{References}

1. Glower DD, Kar S, Trento A, Lim DS, Bajwa T, Quesada R, Whitlow PL, Rinaldi MJ, Grayburn P, Mack MJ, Mauri L, McCarthy PM, Feldman T. Percutaneous mitral valve repair for mitral regurgitation in high-risk patients: results of the EVEREST II study. J Am Coll Cardiol. 2014;64:172-81.

2. Parikh S, Saneto R, Falk MJ, Anselm I, Cohen BH, Haas R, Medicine Society TM. A modern approach to the treatment of mitochondrial disease. Curr Treat Options Neurol. 2009;11:414-30.

3. King DH, Smith EO, Huhta JC, Gutgesell HP. Mitral and tricuspid valve anular diameter in normal children determined by two-dimensional echocardiography. Am J Cardiol. 1985;55:787-9.

4. Dalva M, Bichara GC, Cunha Filho CE, Carneiro GF, Saliba GN, Camacho JA, Zárate JV, Límaco RP. Intermittent annular reduction with Alfieri's repair in the treatment of mitral insufficiency in children: initial results. Rev Bras Cir Cardiovasc. 2009;24:354-8.

\section{Supplementary data}

Moving image 1. 2D TEE biplane image of the left ventricle. ME biplane acquisition showing a modified four-chamber view on the left and a perpendicular LAX view of the LV on the right. Note the enlarged left atrium and the globular LV with apical non-compaction. The MV leaflets are apically tethered during systole and have little opportunity for coaptation. There is posterior and lateral wall akinesis.

Moving image 2. 2D TEE colour flow Doppler of the MV. 2D CFD ME commissural view of the MV showing severe MR originating along the entire coaptation line. 
Moving image 3. 3D colour moving image of the MV with the posteromedial commissure (PMC) on the left and the anterolateral commissure (ALC) towards the right. Unidirectional colour selection was used to demonstrate only regurgitant flow while removing the colour distraction created by mitral inflow. Note that, compared to the orthogonal 2D colour images (Figure 2, Moving image 2), 3D colour is extremely useful in providing details of the geometry of the MR. Two distinct jets are clearly seen: a large medial component and a smaller lateral one. This moving image also shows the aortic valve in front (AV) and moderate TR directed towards the interatrial septum (IAS). Moving image 4. ME CFD biplane image of the MV after deployment of the first MitraClip in the area of A2/P2. The commissural view is seen on the left and a corresponding LAX view of the MV on the right. Note the significant residual MR medial to the first MitraClip.

Moving image 5. ME colour biplane video displaying the MV in a commissural view and a corresponding LAX view after the second MitraClip has been deployed. The moving image displays further reduction of MR leaving only mild to moderate residual MR laterally.

\footnotetext{
The supplementary data are published online at: http://www.pcronline.com/

eurointervention/105th_issue/172
} 